SUPPORTING INFORMATION

\title{
Quantifying Interferent Effects on Molecularly Imprinted Polymer Sensors for Per- and Polyfluoroalkyl Substances (PFAS)
}

Rezvan Kazemi ${ }^{\mathrm{a}}$, Emili I. Potts ${ }^{\mathrm{a}}$, and Jeffrey E. Dick ${ }^{\mathrm{a}, \mathrm{b} *}$

a Department of Chemistry, The University of North Carolina at Chapel Hill, Chapel Hill, NC 27599, USA

${ }^{b}$ Lineberger Comprehensive Cancer Center, School of Medicine, The University of North Carolina at Chapel Hill, Chapel Hill, NC 27599, USA

*To whom correspondence should be addressed: jedick@email.unc.edu 


\section{Table of contents}

Contents

Section S1. Derivation of the binding isotherm equation

Figure S1. DPV responses of MIP in the presence of humic acid

Figure S2. DPV responses of MIP in the presence of chloride ion

Figure S3. DPV responses of MIP in the presence of PFOA

Figure S4. DPV responses of MIP in the presence of PFBS

Section S2. Scatchard plots for a) PFOS b) humic acid c) chloride ion d) PFOA e) PFBS
Page number

S3-S5

S6

S7

S8

S9

S10-S11 


\section{S1. Derivation of binding isotherm equation}

\begin{tabular}{ll}
\hline Acronym & Definition \\
\hline PFOS & Free PFOS molecules \\
BS & Free binding sites \\
PFOS@BS & PFOS bound to BS \\
Rate $_{A}$ & Rate of association process \\
Rate $_{D}$ & Rate of dissociation process \\
$(B S)_{0}$ & Total number of binding sites in the absence of PFOS \\
$(B S)$ & Number of free binding sites in the presence of PFOS \\
{$[X]$} & Concentration representation of X species \\
$i_{0}$ & Experimental current obtained in the absence of PFOS molecules \\
$i_{\text {single } B S}$ & Theoretical current expected from a single binding site \\
$n$ & Number of electrons involved in the electrochemical reaction \\
$D$ & Diffusion coefficient of electroactive species \\
$r$ & Radius of each microelectrode (binding site) radius of each PFOS molecule \\
$C^{*}$ & Bulk concentration of electroactive species
\end{tabular}




$$
\begin{aligned}
& \text { PFOS + BS } \rightleftharpoons \text { PFOS@BS } \\
& \text { Rate }_{A}=k_{A}[\text { PFOS }][B S] \\
& \text { Rate }_{D}=k_{D}[\text { PFOS@BS }]
\end{aligned}
$$

At equilibrium: Rate $_{A}=$ Rate $_{D}$

$\frac{\text { Rate }_{\text {ass. }}}{\text { Rate }_{\text {diss. }}}=\frac{[P F O S @ B S]}{[P F O S][B S]}=K_{A}$

$[B S]_{0}=[B S]+[P F O S @ B S]$

$[B S]_{0}=\frac{[P F O S @ B S]}{[P F O S] K_{A}}+[P F O S @ B S]=[P F O S @ B S]\left(\frac{1+[P F O S] K_{A}}{[P F O S] K_{A}}\right)$

$[P F O S @ B S]=\frac{(P F O S @ B S)}{V_{\text {polymer } \times N_{A}}}=\frac{[B S]_{0}[P F O S] K_{A}}{1+[P F O S] K_{A}}$

$(P F O S @ B S)=\frac{(B S)_{0}[P F O S] K_{A}}{1+[P F O S] K_{A}}$

Assuming each binding site as a disk microelectrode, the limiting current at each binding site can be calculated from Equation (9), where $n$ is the number of electrons involved in the electrochemical reaction, $F$ is the Faraday constant, $D$ is the diffusion coefficient of electroactive species $(\mathrm{FcCOOH}), C^{*}$ is the bulk concentration of electroactive species, $r$ is the radius of binding sites. Assuming each binding site has the same length as a PFOS molecule, $r$ can be estimated from the radius of a PFOS molecule $\left(8.55 \times 10^{-8} \mathrm{~cm}\right)$.

$$
\begin{aligned}
& i_{\text {single BS }} \sim i_{\text {microelectrode }}=4 n F D C^{*} r \\
& =4 \times 1 \times 96485 \mathrm{C} . \mathrm{mol}^{-1} \times 1.9 \times 10^{-5} \mathrm{~cm}^{2} . \mathrm{s}^{-1} \times 0.5 \times 10^{-6} \mathrm{~mol} . \mathrm{cm}^{-3} \times 8.55 \times 10^{-8} \mathrm{~cm} \\
& =3.13 \times 10^{-13} \mathrm{~A}
\end{aligned}
$$

Therefore, the total current in the absence of PFOS (io) and in the presence of PFOS molecules (i) can be estimated from the following equations:

$i_{0}=(B S)_{0} \times i_{\text {single } B S}=(B S)_{0} \times 4 n F D C^{*} r$

$i=(B S) \times i_{\text {single } B S}=(B S) \times 4 n F D C^{*} r$

Where $(B S)_{0}$ is the total number of binding sites inside the polymer film in the absence of PFOS molecules, and (BS) is the free binding sites in the presence of PFOS molecules. 
The current decrease in the presence of PFOS molecules (io-i) can be estimated from the following equation:

$i_{0}-i=\left((B S)_{0}-(B S)\right) \times 4 n F D C^{*} r=(P F O S @ B S) \times 4 n F D C^{*} r$

Substituting Equation 8 into Equation 12 gives the Langmuir expression of our model:

$i_{0}-i=\frac{(B S)_{0}[P F O S] K_{A}}{1+[P F O S] K_{A}} \times i_{\text {single } B S}=\frac{(B S)_{0}[P F O S] K_{A}}{1+[P F O S] K_{A}} \times 4 n F D C^{*} r$

The number of binding sites can be estimated from the ratio between the total current obtained from MIP in the absence of PFOS (io) and the expected current from each binding site ( $i_{\text {single } B S}$ ):

$(B S)_{0}=\frac{i_{0}}{i_{\text {single } B S}}$ 


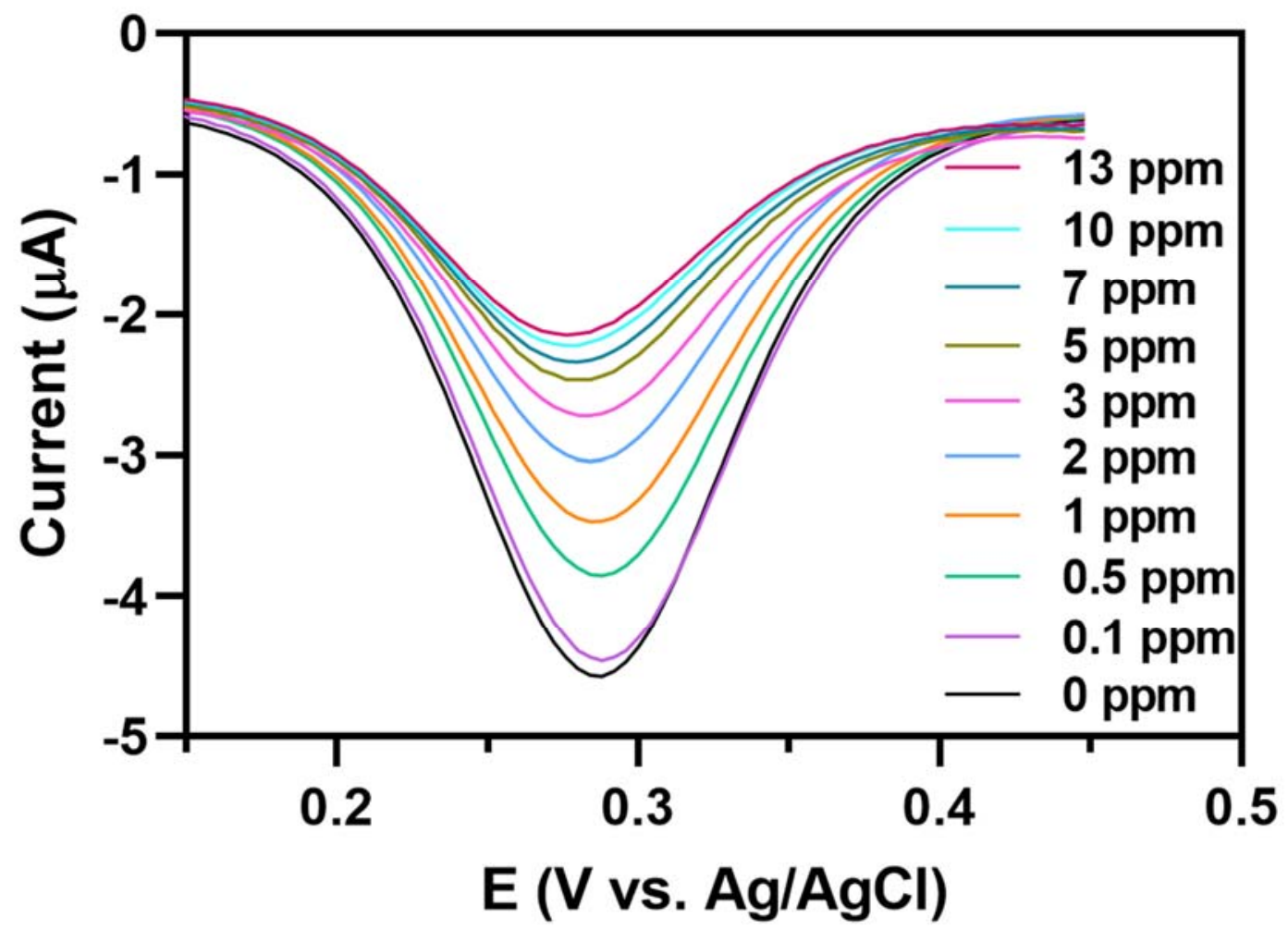

Figure S2. DPVs of $0.5 \mathrm{mM} \mathrm{FcCOOH}$ in $0.01 \mathrm{M}$ ammonia buffer ( $\mathrm{pH} 8.4$ ) obtained at MIP electrode after 15 min incubation in the solution containing humic acid at different concentrations mentioned in the figure. 


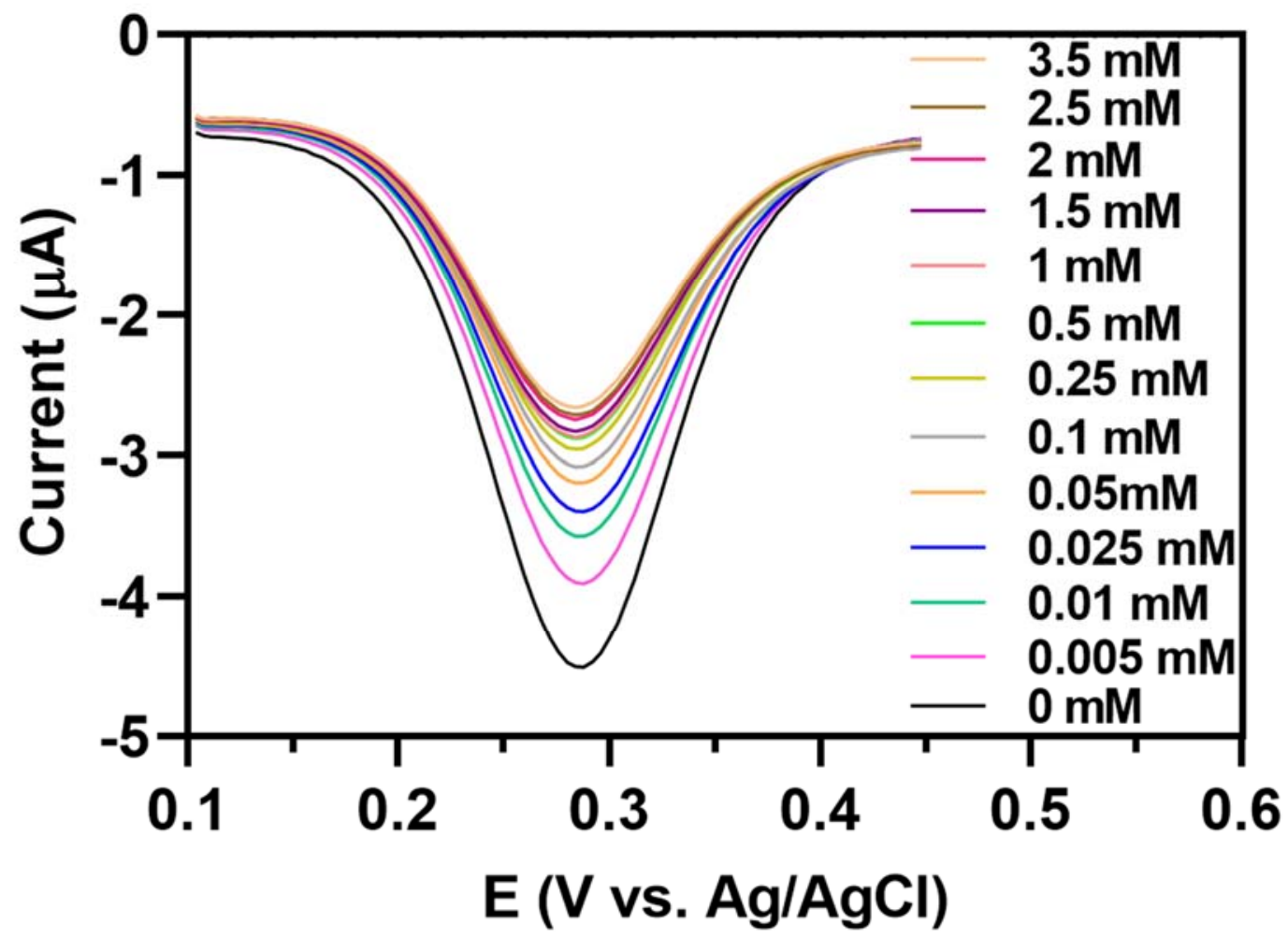

Figure S3. DPVs of $0.5 \mathrm{mM} \mathrm{FcCOOH}$ in $0.01 \mathrm{M}$ ammonia buffer ( $\mathrm{pH} 8.4$ ) obtained at MIP electrode after 15 min incubation in the solution containing sodium chloride at different concentrations mentioned in the figure. 


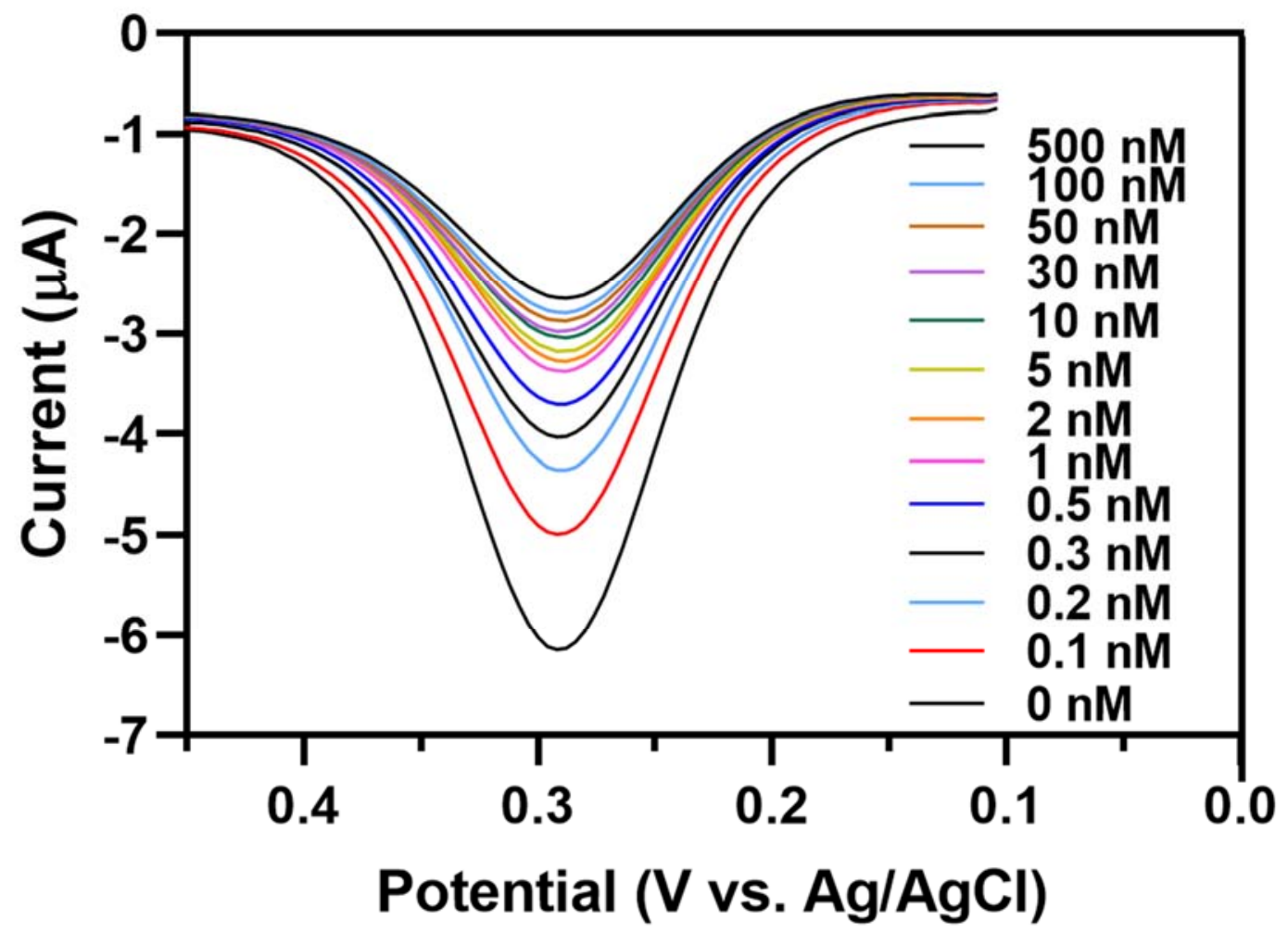

Figure S4. DPVs of $0.5 \mathrm{mM} \mathrm{FcCOOH}$ in $0.01 \mathrm{M}$ ammonia buffer ( $\mathrm{pH} 8.4$ ) obtained at MIP electrode after 15 min incubation in the solution containing PFOA at different concentrations mentioned in the figure. 


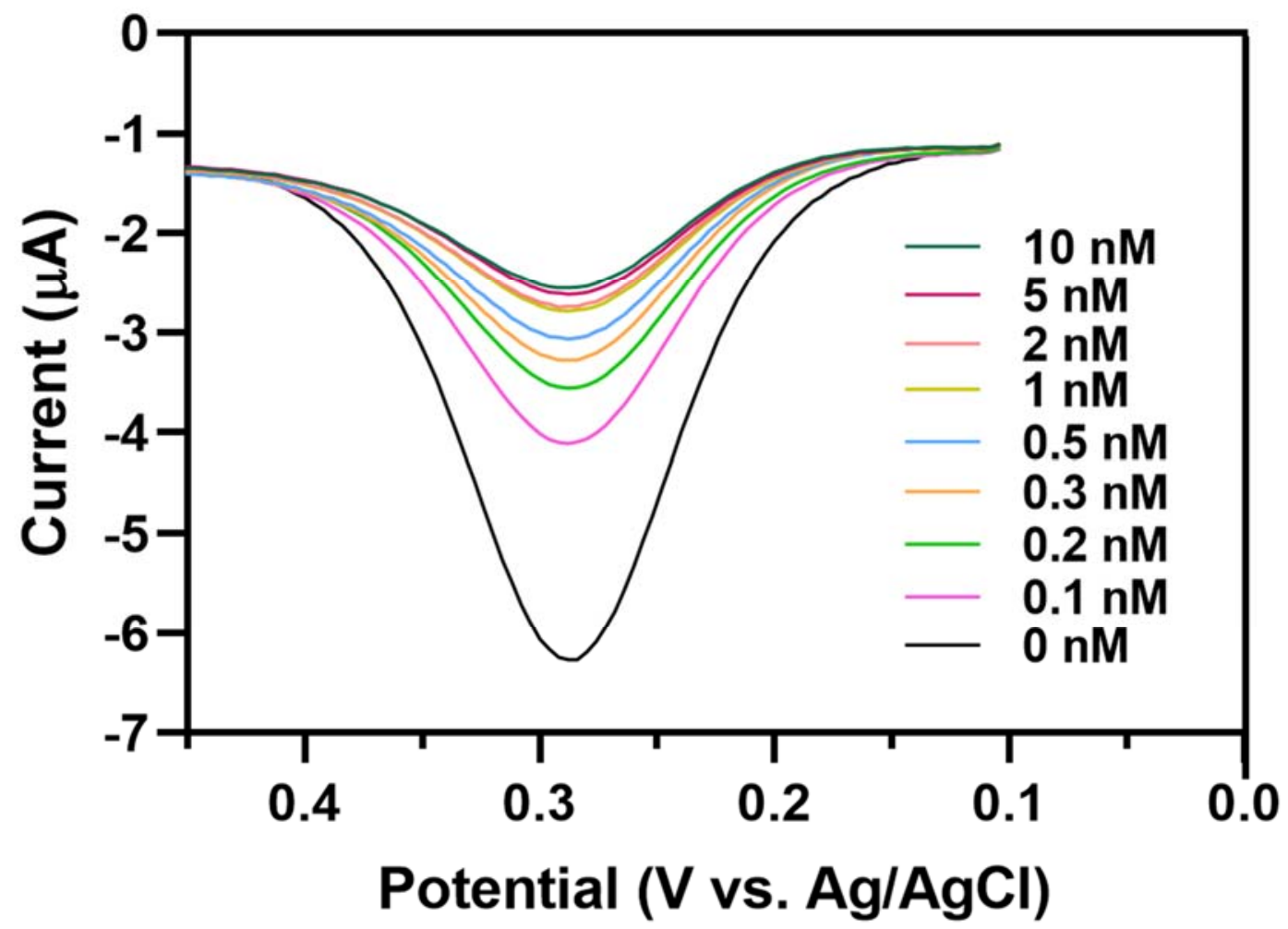

Figure S5. DPVs of $0.5 \mathrm{mM} \mathrm{FcCOOH}$ in $0.01 \mathrm{M}$ ammonia buffer ( $\mathrm{pH} 8.4$ ) obtained at MIP electrode after 15 min incubation in the solution containing PFBS at different concentrations mentioned in the figure. 
S6. Scatchard expression of Langmuir equation

The general form of Langmuir equation and its Scatchard expression are given by:

Langmuir equation: $B=\frac{(B S)_{0} K_{A} F}{1+K_{A} F}$

Scatchard expression: $\frac{B}{F}=K_{A}(B S)_{0}-K_{A} B$

Where $B$ and $F$ is the amount of bound and free analyte, $K_{A}$ is the association constant, and $(B S)_{0}$ is the total number of the binding sites in the MIP. The electrochemical expression of Langmuir aquation for our model is given by:

$$
i_{0}-i=\frac{(B S)_{0}[P F O S] K_{A}}{1+[P F O S] K_{A}} \times i_{\text {single } B S}=\frac{(B S)_{0}[P F O S] K_{A}}{1+[P F O S] K_{A}} \times 4 n F D C^{*} r
$$

Where $\left(i_{0}-i\right)$ is electrochemically equivalence to $B$, and $i_{\text {single } B S}$ can be considered the electrochemical proportionality constant. The Scatchard expression of Equation (17) is given by:

$\frac{i_{0}-i}{[P F O S]}=\left(K_{A}(B S)_{0} i_{\text {single } B S}\right)-K_{A}\left(i_{0}-i\right)=\left(K_{A}(B S)_{0} 4 n F D C^{*} r\right)-K_{A}\left(i_{0}-i\right)$

Ploting $\frac{i_{0}-i}{[P F O S]}$ vs. $\left(i_{0}-i\right)$ gives a line with a slope of $-K_{A}$ and $y$-intercept of $\left(K_{A}(B S)_{0} 4 n F D C^{*} r\right)$. 

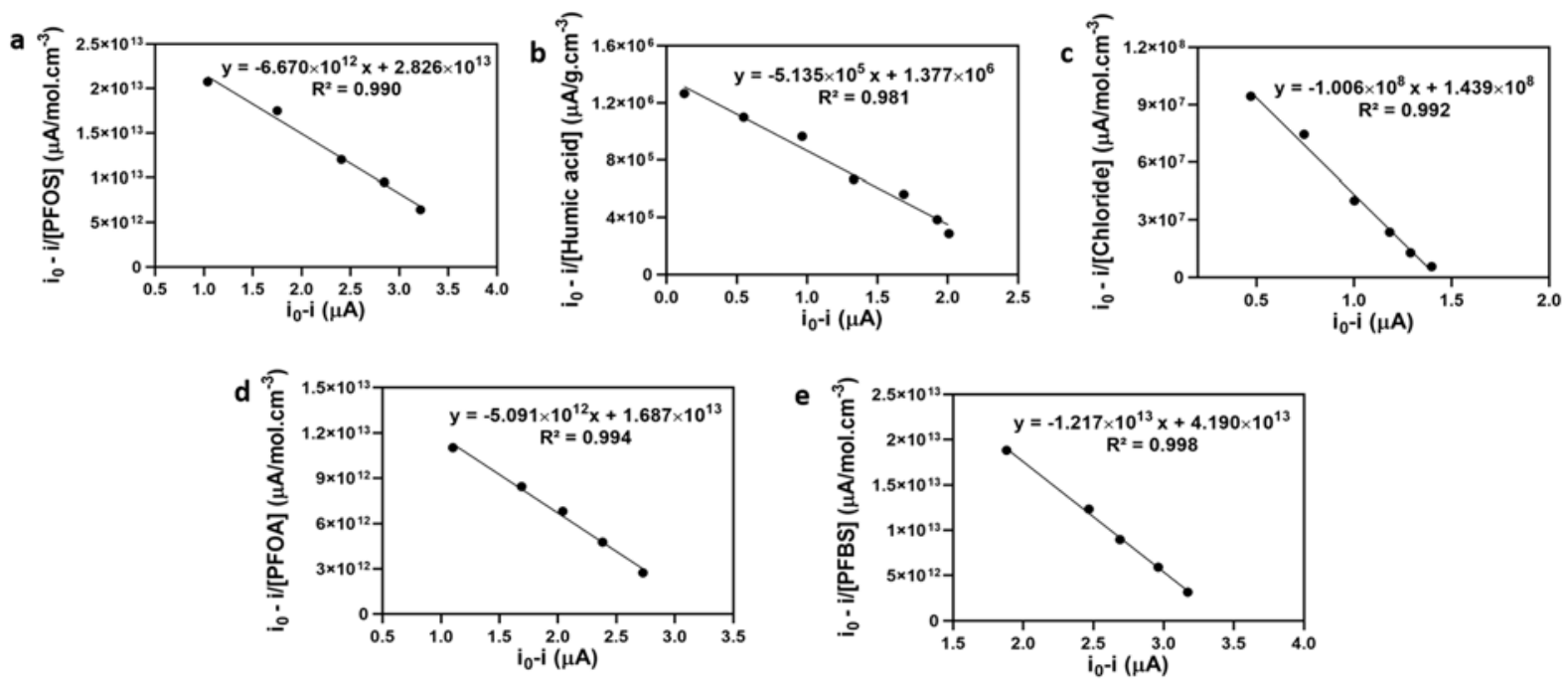

Figure S6. Scatchard plot of corresponding DPV peak current vs. concentration of analyte represented in the manuscript for a) PFOS, b) humic acid, c) chloride, d) PFOA, and e) PFBS 